\title{
AMENDMENTS
}

\section{Author Correction: A co-clinical approach identifies mechanisms and potential therapies for androgen deprivation resistance in prostate cancer}

Andrea Lunardi, Ugo Ala, Mirjam T. Epping, Leonardo Salmena, John G. Clohessy, Kaitlyn A. Webster, Guocan Wang, Roberta Mazzucchelli, Maristella Bianconi, Edward C. Stack, Rosina Lis, Akash Patnaik, Lewis C. Cantley, Glenn Bubley, Carlos Cordon-Cardo, William L. Gerald, Rodolfo Montironi, Sabina Signoretti, Massimo Loda, Caterina Nardella and

Pier Paolo Pandolfi

Correction to: Nature Genetics https://doi.org/10.1038/ng.2650, published online 2 June 2013.

In the version of this article initially published, the Pbsn-cre4; Pten ${ }^{f l f l}$; Trp5 $3^{f l / f l}$ and Pbsn-cre4; Pten flffl Zbtb7a $a^{f l f l}$ labels above the protein blot images in Fig. 3c were inadvertently switched.

In the legend of Fig. 4d, 'Protein blot analysis on the samples in b' should have read 'Protein blot analysis on the samples in Fig. 3c'.

In Supplementary Fig. 1e, the images labeled as AR-stained vehicle-treated control Pbsn-cre4; Pten flff; Zbtb7a $a^{f l f l}$ (chemical castration) inadvertently showed control non-castrated Pbsn-cre4; Pten ${ }^{f l f l} ; Z b t b 7 a^{f l / f l}$ prostate tumor images from mice in Fig. $3 \mathrm{~d}$. The original and corrected images are shown in the correction notice.
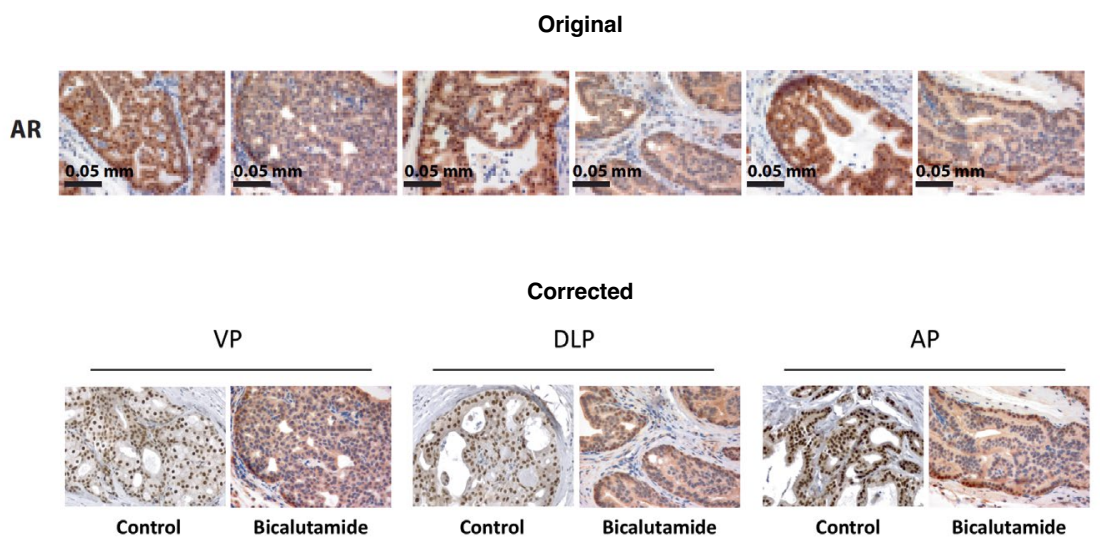

Supplementary Fig. 1e I Original and corrected.

Published online: 3 September 2020

https://doi.org/10.1038/s41588-020-0701-7

(C) The Author(s), under exclusive licence to Springer Nature America, Inc. 2020

\section{Author Correction: Exploring the coronavirus pandemic with the WashU Virus Genome Browser}

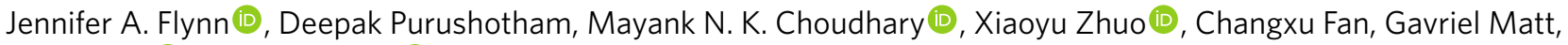
Daofeng Li (iD) and Ting Wang (i)

Correction to: Nature Genetics https://doi.org/10.1038/s41588-020-0697-Z, published online 9 September 2020.

In the version of this article originally published, the name of author Xiaoyu Zhuo was incorrectly spelled as Xiaoyu Zhou. The error has been corrected in the HTML and PDF versions of the article.

Published online: 16 September 2020

https://doi.org/10.1038/s41588-020-00714-w

(C) The Author(s), under exclusive licence to Springer Nature America, Inc. 2020 